\title{
Melioidosis en Colombia, descripción de un caso clínico y consideraciones epidemiológicas
}

José Y. Rodríguez¹, Carlos A. Álvarez-Moreno ${ }^{2,3}$, Jorge A. Cortés², Gerson J. Rodríguez ${ }^{1}$, Kelin Esquea ${ }^{3}$, Heidy Pinzón ${ }^{4}$, María J. Mendoza ${ }^{1}$, Yiceth Acosta ${ }^{5}$

${ }^{1}$ Centro de Investigaciones Microbiológicas del Cesar, Valledupar, Colombia

${ }^{2}$ Facultad de Medicina, Universidad Nacional de Colombia, Bogotá, D.C., Colombia

${ }^{3}$ Clínica Universitaria Colombia, Clínicas Colsanitas, Bogotá, D.C., Colombia

${ }^{4}$ Clínica Laura Daniela, Valledupar, Colombia

${ }^{5}$ Clínica Médicos SA, Valledupar, Colombia

La melioidosis es una enfermedad infecciosa causada por Burkholderia pseudomallei cuyo diagnóstico clínico puede ser difícil debido a su variada presentación clínica y a las dificultades del diagnóstico microbiológico, por lo cual pueden requerirse técnicas moleculares para su adecuada identificación una vez se sospecha su presencia.

Son pocos los antibióticos disponibles para el tratamiento de esta enfermedad y, además, deben usarse durante un tiempo prolongado. Aunque se conoce por ser endémica en Tailandia, Malasia, Singapur, Vietnam y Australia, en Colombia se han reportado algunos pocos casos.

Se presenta un caso de melioidosis en la región norte de Colombia, se hace una revisión de las características clínicas y el tratamiento, y se describe la epidemiología local de esta enfermedad.

Palabras clave: Burkholderia pseudomallei; melioidosis/epidemiología; informes de caso; Colombia.

\section{Melioidosis in Colombia, description of a clinical case and epidemiological considerations}

Melioidosis is an infectious disease caused by Burkholderia pseudomallei whose clinical diagnosis can be difficult due not only to its varied clinical presentation but also to the difficulties in the microbiological diagnosis. Thus, it may be necessary to use molecular techniques for its proper identification once it is suspected.

Recibido: 08/06/18

Correspondencia:

José Y. Rodríguez, Centro de Investigaciones Microbiológicas del Cesar, Calle 16C N 19D-14, Barrio Dangond, Valledupar, Colombia

Teléfono: (0575) 5800126 - (0575) 5806696 jyrodriguezq@gmail.com

Contribución de los autores:

José Y. Rodríguez y Gerson J. Rodríguez: manejo clínico de los pacientes

Kelin Esquea, Heidy Pinzón, Yiceth Acosta y María J.

Mendoza: identificación de microorganismos

José Y. Rodríguez, Gerson J. Rodríguez, Carlos A.

Álvarez-Moreno y Jorge A. Cortés: análisis de los

datos clínicos, microbiológicos y moleculares

Todos los autores participaron en la escritura del

manuscrito.

Financiación

No hubo fuentes de financiación.

Conflicto de intereses:

Los autores declaran no tener conflictos de intereses.
There are few antibiotics available for the treatment of this disease, which must be used over a long period of time. Although it is known to be endemic in Thailand, Malaysia, Singapore, Vietnam, and Australia, in Colombia there are few reported cases.

We describe a case of melioidosis in the northern region of Colombia. Additionally, we review its clinical characteristics and treatment and we describe the local epidemiology of this disease.

Keywords: Burkholderia pseudomallei; melioidosis/epidemiology; case reports; Colombia.

La melioidosis es una enfermedad infecciosa causada por Burkholderia pseudomallei, una bacteria saprofita del suelo (1). Esta enfermedad tiene una amplia gama de presentaciones clínicas, algunas con altas tasas de mortalidad. La melioidosis es un problema de salud pública en áreas del sudeste asiático y Australia, y se considera una enfermedad infecciosa emergente en muchos países tropicales en desarrollo, incluido Colombia (2). A continuación, se presenta un caso y se describen la epidemiología local, las características clínicas y el tratamiento de la enfermedad.

\section{Reporte de caso}

Se trata de un hombre de 56 años de edad procedente de La Loma (zona rural del municipio de El Paso, Cesar, Colombia), con antecedentes de diabetes mellitus de tipo 2 e insuficiencia renal crónica bajo tratamiento médico.

Consultó por presentar síntomas urinarios de una semana de evolución. En el momento del ingreso, tenía una temperatura de $39,5^{\circ} \mathrm{C}$ y refirió sentir dolor en la percusión con el puño en ambas fosas renales. 
Los exámenes de laboratorio evidenciaron leucocitosis con anemia (leucocitos: 11,000 células/ $\mu$ l, neutrófilos: $77 \%$, hemoglobina: 7,9 $\mathrm{g} / \mathrm{dl}$, plaquetas: 437.000 por $\mu \mathrm{l}$ ), elevación de azoados (creatinina: $2,0 \mathrm{mg} / \mathrm{dl}$, nitrógeno ureico en sangre: $31 \mathrm{mg} / \mathrm{dl}$ ) e hiperglucemia (glucosa: $176 \mathrm{mg} / \mathrm{dl}$ ). En la ecografía renal se observó hidronefrosis bilateral.

Se inició tratamiento empírico con 2,25 g de piperacilina y tazobactam intravenosos cada seis horas. Los resultados de los hemocultivos fueron negativos en el quinto día, en tanto que el urocultivo fue positivo para $B$. pseudomallei, razón por la cual se decidió cambiar el tratamiento antibiótico a $500 \mathrm{mg}$ de meropenem intravenoso cada ocho horas durante diez días.

El paciente presentó una adecuada evolución clínica, por lo que se decidió continuar el manejo de forma ambulatoria con 160/800 mg de trimetoprim-suffametoxazol cada 12 horas por vía oral durante tres meses.

Siete meses después, el paciente reingresó por infección necrosante del pie derecho asociada con fiebre alta no cuantificada, vómito en múltiples oportunidades y un episodio convulsivo. Los familiares refirieron que el paciente no se había tomado el tratamiento con trimetoprim-suffametoxazol en la forma indicada.

Los exámenes de laboratorio evidenciaron leucocitosis y neutrofilia con anemia (leucocitos: 17.900 células/ $\mu$ l, neutrófilos: $93 \%$, hemoglobina: 7,5 g/dl), acidosis metabólica grave $\left(\mathrm{pH}: 7,328, \mathrm{PCO}_{2}: 18 \mathrm{mmol}, \mathrm{HCO}_{3}: 9,2 \mathrm{mmol}\right.$; base exceso: -14,2) y elevación de azoados (creatinina: $8,9 \mathrm{mg} / \mathrm{dll}$, nitrógeno ureico en sangre: $96 \mathrm{mg} / \mathrm{dl}$ ).

El paciente fue trasladado a la unidad de cuidados intensivos para monitorear su estado hemodinámico, administrarle agentes vasopresores e iniciar hemodiafiltración venovenosa continua.

Dado el antecedente de melioidosis, se decidió iniciar el tratamiento antibiótico intravenoso con $1 \mathrm{~g}$ de ceftazidima y 160/800 mg de trimetoprimsuffametoxazol cada ocho horas.

Durante la hospitalización, el paciente fue sometido a procedimientos quirúrgicos y se le amputó la falange proximal del pie derecho. Los hemocultivos, el urocultivo y el cultivo de la secreción del pie derecho fueron positivos para B. pseudomallei.

El paciente fue dado de alta después de 35 días de hospitalización y múltiples lavados quirúrgicos, y se le continuó administrando un tratamiento oral de consolidación con 160/800 mg de trimetoprim-suffametoxazol cada 12 horas y $100 \mathrm{mg}$ de doxiciclina cada 12 horas, durante seis meses.

Para la identificación microbiológica, se utilizó el sistema MicroScan WalkAway ${ }^{\mathrm{TM}}$ (Beckman Coulter, USA); la posterior identificación molecular permitió obtener la secuencia, la cual se ingresó en GenBank (Burkholderia pseudomallei strain CIMCE 006 16S ribosomal RNA gene, partial sequence. Accession: KY996759). A los seis meses del egreso hospitalario, el paciente no había presentado recurrencias de la enfermedad.

\section{Discusión y revisión de la literatura}

En 1992, se definió el género Burkholderia como separado del género Pseudomonas con base en las secuencias del ARN ribosómico 16S (rRNA 16S), por homología de sus valores de ADN, su composición de lípidos 
celulares y ácidos grasos, y sus características fenotípicas (3). En este género, con más de 100 especies, B. pseudomallei (agente etiológico de la melioidosis), B. mallei (agente etiológico del muermo) y el complejo B. cepacia (compuesto, por lo menos, por 20 especies y causante de infecciones hospitalarias y pulmonares oportunistas), son las especies generalmente asociadas con la infección en seres humanos (4-6).

La melioidosis se ha descrito como endémica en el sudeste y el sur de Asia y en el norte de Australia $(7,8)$. Se estima que hay 165.000 casos al año en todo el mundo, de los cuales 89.000 resultan fatales $(2,9)$, y se la reconoce como una de las principales causas de muerte por neumonía y sepsis en Tailandia, Malasia, Singapur, Vietnam y Australia (10).

La aparición de B. pseudomallei en los Estados Unidos se ha asociado con la trata de esclavos en los siglos XVIII y XIX, dada la similitud genética entre aislamientos de África y América que sugiere que su origen se dio a partir de su introducción en el territorio continental entre 1759 y 1806 (8). Si esta teoría es correcta, la diseminación debió extenderse por toda América, especialmente en los países de habla inglesa, francesa y portuguesa, y en menor medida, en los países de habla hispana, pues los españoles limitaron el esclavismo en comparación con otros grupos de comerciantes de la época (11); además, esto coincide con los lugares de detección de casos en el continente.

Se ha presentado un incremento del número de casos por fuera de la región Asia-Pacífico, incluido el continente americano, aunque no está claro si dicho incremento refleja un aumento de la incidencia o el fortalecimiento de los laboratorios de microbiología y de los programas de búsqueda y vigilancia de esta enfermedad (12). Una revisión de los casos registrados en América entre 1947 y 2015, los ubica fundamentalmente en Centroamérica y el Caribe (41 de 95) y en Suramérica, especialmente en Brasil (28 de 95) (1).

En Colombia, la melioidosis no es una enfermedad de interés en salud pública (vigilancia activa). Sin embargo, después de Brasil, es el segundo país suramericano con más casos reportados en la literatura médica (1). El número real de casos se desconoce, probablemente como consecuencia del desconocimiento de la enfermedad por parte de los profesionales de la salud, lo que desemboca en la falta de sospecha diagnóstica. Ello se suma a la ausencia de la tecnología necesaria para su diagnóstico adecuado y al hecho de que no se reportan los casos diagnosticados, aunque en los artículos consultados se describen, por lo menos, 12 casos de melioidosis en el país (13-18).

En Valledupar (Cesar), ciudad ubicada en el norte del país (a $180 \mathrm{~km}$, aproximadamente, de la línea de costa), se viene haciendo búsqueda activa de casos de melioidosis desde el 2014 y se han encontrado 15 casos (cuadro 1) provenientes de los departamentos de Cesar, Bolívar y Magdalena, los cuales comparten el territorio de la Sierra Nevada de Santa Marta, macizo montañoso aislado de la cordillera de los Andes, y forman parte de la región natural conocida como la depresión momposina (figura 1). Esta región se caracteriza por una elevada humedad debida a los grandes volúmenes de agua con los que cuenta permanentemente y a promedios de precipitación anual entre los 1.500 y los $2.000 \mathrm{~mm}$.

La melioidosis afecta principalmente a personas que tienen contacto frecuente con la tierra y el agua contaminadas con B. pseudomallei. Las principales rutas de infección son la inoculación, la inhalación y la ingestión. Su presentación clínica, gravedad y resultados dependen de la carga bacteriana 
Cuadro 1. Características epidemiológicas y demográficas, tratamiento antibiótico y resultados clínicos de los pacientes con melioidosis en Cesar

\begin{tabular}{|c|c|c|c|c|c|c|c|c|c|c|}
\hline Paciente & $\begin{array}{l}\text { Fecha de } \\
\text { ingreso }\end{array}$ & Edad & Sexo & Antecedentes & Presentación & Tipo de muestra & Tratamiento & $\mathrm{UCl}$ & Resultado & Recurrencia \\
\hline 1 & 03/09/2014 & 72 & $\mathrm{M}$ & $\begin{array}{l}\text { Diabetes mellitus de } \\
\text { tipo 2; hipertensión } \\
\text { arterial }\end{array}$ & $\begin{array}{l}\text { Neumonía con } \\
\text { bacteriemia }\end{array}$ & $\begin{array}{l}\text { Hemocultivo, } \\
\text { urocultivo y } \\
\text { secreción } \\
\text { orotraqueal }\end{array}$ & $\begin{array}{l}\text { I: meropenem C: } \\
\text { TMP-SMX }\end{array}$ & Sí & Fallecido & -- \\
\hline 2 & 09/08/2015 & 60 & $\mathrm{~F}$ & $\begin{array}{l}\text { Diabetes mellitus de } \\
\text { tipo 2; hipertensión } \\
\text { arterial }\end{array}$ & $\begin{array}{l}\text { Infección de } \\
\text { piel y tejidos } \\
\text { blandos y } \\
\text { bacteriemia }\end{array}$ & $\begin{array}{l}\text { Secreción de } \\
\text { herida y } \\
\text { hemocultivos }\end{array}$ & $\begin{array}{l}\text { I: meropenem } \\
\text { C: TMP-SMX }\end{array}$ & No & Vivo & No \\
\hline $3^{*}$ & $31 / 05 / 2016$ & 49 & $\mathrm{M}$ & $\begin{array}{l}\text { Diabetes mellitus de } \\
\text { tipo 2; enfermedad } \\
\text { de Hansen: eritema } \\
\text { nudoso leproso, uso } \\
\text { de esteroides }\end{array}$ & $\begin{array}{l}\text { Neumonía con } \\
\text { bacteriemia }\end{array}$ & Hemocultivo & $\begin{array}{l}\text { I: meropenem } \\
\text { C: TMP/SMX }\end{array}$ & No & Vivo & No \\
\hline $4^{*}$ & $24 / 10 / 2016$ & 71 & $\mathrm{M}$ & $\begin{array}{l}\text { Hipertensión arterial, } \\
\text { mieloma múltiple, } \\
\text { trasplante de medula } \\
\text { ósea, uso de talidomida } \\
\text { y esteroides }\end{array}$ & $\begin{array}{l}\text { Neumonía con } \\
\text { bacteriemia }\end{array}$ & Hemocultivo & $\begin{array}{l}\text { I: meropenem } \\
\text { C: TMP/SMX }\end{array}$ & No & Vivo & No \\
\hline 5 & 19/11/2016 & 66 & $\mathrm{M}$ & $\begin{array}{l}\text { Diabetes mellitus de } \\
\text { tipo } 2 \text {; hipertensión } \\
\text { arterial }\end{array}$ & $\begin{array}{l}\text { Neumonía con } \\
\text { bacteriemia }\end{array}$ & $\begin{array}{l}\text { Hemocultivo y } \\
\text { secreción } \\
\text { orotraqueal }\end{array}$ & PIP-TAZ & Sí & Fallecido & -- \\
\hline $6^{*}$ & $05 / 12 / 2016$ & 56 & $\mathrm{M}$ & $\begin{array}{l}\text { Diabetes mellitus de } \\
\text { tipo } 2\end{array}$ & $\begin{array}{l}\text { Infección de } \\
\text { vías urinarias }\end{array}$ & Urocultivo & $\begin{array}{l}\text { I: meropenem } \\
\text { C: TMP-SMX }\end{array}$ & No & Vivo & $\begin{array}{l}\text { A los } 6 \text { meses: } \\
\text { infección de } \\
\text { vías urinarias, } \\
\text { bacteriemia e } \\
\text { infección de piel } \\
\text { y tejidos blandos }\end{array}$ \\
\hline $7^{*}$ & 19/12/2016 & 54 & $\mathrm{M}$ & $\begin{array}{l}\text { Posoperatorio de } \\
\text { osteosíntesis de tibia } \\
\text { y peroné por fractura } \\
\text { abierta }\end{array}$ & $\begin{array}{l}\text { Infección de } \\
\text { piel y tejidos } \\
\text { blandos }\end{array}$ & $\begin{array}{l}\text { Secreción de } \\
\text { herida }\end{array}$ & $\begin{array}{l}\text { I: meropenem } \\
\text { C: TMP-SMX }\end{array}$ & No & Vivo & No \\
\hline $8^{*}$ & $22 / 04 / 2017$ & 51 & $\mathrm{~F}$ & $\begin{array}{l}\text { Diabetes mellitus de } \\
\text { tipo } 2\end{array}$ & Neumonía & $\begin{array}{l}\text { Cultivo de esputo } \\
\text { y } \\
\text { lavado } \\
\text { bronquioalveolar }\end{array}$ & $\begin{array}{l}\text { I: ceftazidima } \\
\text { C: TMP-SMX }\end{array}$ & No & Vivo & No \\
\hline 9 & $13 / 05 / 2017$ & $\begin{array}{c}3 \\
\text { meses }\end{array}$ & $\mathrm{F}$ & $\begin{array}{l}\text { Prematuro de } 34 \\
\text { semanas }\end{array}$ & $\begin{array}{l}\text { Infección de } \\
\text { vías urinarias }\end{array}$ & Urocultivo & Meropenem & No & Vivo & No \\
\hline 10 & 13/06/2017 & 47 & $\mathrm{M}$ & $\begin{array}{l}\text { Posoperatorio de } \\
\text { lavado quirúrgico y } \\
\text { desbridamiento por } \\
\text { heridas complejas en } \\
\text { rodilla debidas a trauma }\end{array}$ & $\begin{array}{l}\text { Infección de } \\
\text { piel y tejidos } \\
\text { blandos }\end{array}$ & $\begin{array}{l}\text { Secreción de } \\
\text { herida }\end{array}$ & $\begin{array}{l}\text { I: Meropenem } \\
\text { C: TMP-SMX }\end{array}$ & No & Vivo & No \\
\hline 11 & $16 / 06 / 2017$ & 79 & M & Ninguno & $\begin{array}{l}\text { Neumonía con } \\
\text { bacteriemia }\end{array}$ & Hemocultivo & PIP-TAZ & Sí & Fallecido & -- \\
\hline 12 & $20 / 06 / 2017$ & 36 & $\mathrm{M}$ & $\begin{array}{l}\text { Diabetes mellitus, } \\
\text { hipertensión arterial }\end{array}$ & $\begin{array}{l}\text { Neumonía con } \\
\text { bacteriemia }\end{array}$ & Hemocultivo & $\begin{array}{l}\text { I: ceftazidima } \\
\text { C: TMP-SMX }\end{array}$ & Sí & Vivo & No \\
\hline $13^{*}$ & $10 / 11 / 2017$ & 3 & $\mathrm{~F}$ & Ninguno & $\begin{array}{l}\text { Neumonía con } \\
\text { bacteriemia }\end{array}$ & $\begin{array}{l}\text { Hemocultivo, } \\
\text { secreción } \\
\text { orotraqueal }\end{array}$ & $\begin{array}{l}\text { Ceftriaxona más } \\
\text { clindamicina más } \\
\text { claritromicina }\end{array}$ & Sí & Fallecido & -- \\
\hline 14 & $01 / 01 / 2018$ & 89 & $\mathrm{~F}$ & Ninguno & $\begin{array}{l}\text { Neumonía con } \\
\text { bacteriemia }\end{array}$ & $\begin{array}{l}\text { Hemocultivo, } \\
\text { secreción } \\
\text { orotraqueal }\end{array}$ & PIP-TAZ & Sí & Fallecido & -- \\
\hline 15 & 08/04/2018 & 58 & $\mathrm{M}$ & $\begin{array}{l}\text { Secuelas de trauma } \\
\text { cráneoencefálico, } \\
\text { paciente con } \\
\text { traqueotomía }\end{array}$ & $\begin{array}{l}\text { Neumonía con } \\
\text { bacteriemia }\end{array}$ & Hemocultivo & $\begin{array}{l}\text { I: ceftazidima } \\
\text { C: TMP-SMX y } \\
\text { doxiciclina }\end{array}$ & Sí & Vivo & No \\
\hline
\end{tabular}

UCI: unidad de cuidados intensivos; I: inducción; C: consolidación; TMP/SMX: trimetoprim-suffametoxazol; PIP/TAZ: piperacilina-tazobactam

* Identificación del microorganismo mediante biología molecular 


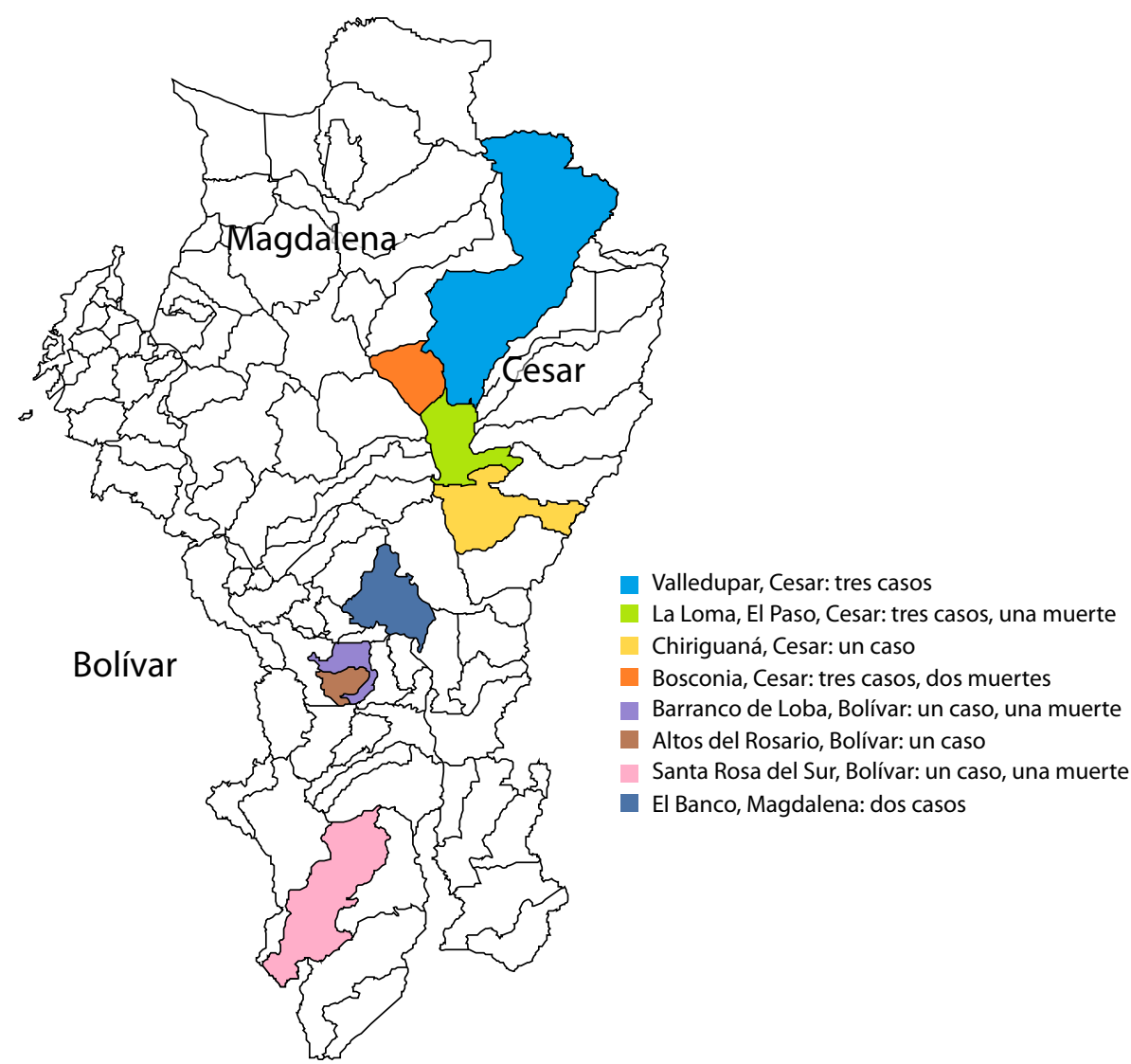

Figura 1. Procedencia y desenlace de los 15 casos de melioidosis diagnosticados en el departamento del Cesar (2014-2018)

en el momento de la exposición, la ruta de infección (inhalatoria o percutánea), la virulencia de la cepa y el estado inmunológico del individuo (19).

Entre los principales factores de riesgo de la enfermedad, se han descrito la diabetes mellitus, la enfermedad pulmonar crónica, la enfermedad renal crónica, el abuso de alcohol, la talasemia, el cáncer, el tratamiento con esteroides y otros tratamientos inmunosupresores $(19,20)$.

Los casos de melioidosis suelen ser producto de una infección adquirida durante la temporada de lluvias y el periodo de incubación promedio es de nueve días (rango: 1 a 21 días) $(21,22)$. El $4 \%$ de los casos corresponde a la reactivación de un foco latente, y clínicamente se presenta en forma localizada o diseminada (19). Por su ubicación cerca de la línea del ecuador, en Colombia no hay estaciones sino temporadas de lluvia que alternan con temporadas secas. Además, algunas zonas del país presentan condiciones similares a las de los nichos ecológicos de la enfermedad en el sureste asiático y, también, cuentan con cultivos de arroz o algodón.

Los estudios serológicos demuestran que la mayoría de los pacientes con infección por B. pseudomallei son asintomáticos (23). La melioidosis puede presentar un amplio rango de presentaciones clínicas que simulan otras enfermedades, como el cáncer o la tuberculosis (24).

La presentación clínica más común es la neumonía (36 a 51 \%), seguida por infecciones de la piel y de los tejidos blandos (13 a $33 \%$ ), infecciones 
genitourinarias (7 a $14 \%$ ), bacteriemia primaria (10 a $15 \%$ ), e infecciones osteoarticulares (4\%) y neurológicas (3 a $7 \%$ ).

En más del $80 \%$ de los casos, su presentación es aguda (menos de dos meses de evolución de los síntomas) y la neumonía se asocia con la mayor tasa de mortalidad (hasta $63 \%$ ), tres veces más que el resto de presentaciones clínicas $(20,22)$.

La melioidosis crónica (enfermedad de más de dos meses de duración) se presenta usualmente en forma de neumonía crónica (que imita la tuberculosis) o de úlceras o abscesos de piel y tejidos blandos (19). Se han reportado diferencias regionales en la presentación clínica, por ejemplo, abscesos parotídeos en $40 \%$ de los niños tailandeses, abscesos prostáticos hasta en $20 \%$ de los hombres australianos o encefalitis con compromiso del tallo cerebral en $4 \%$ de los casos en el norte de Australia (19).

La recurrencia de esta enfermedad puede presentarse en 5 a $25 \%$ de los casos y debe considerarse en pacientes con antecedentes de melioidosis que hayan consultado por un episodio febril $(20,25)$. Esta presentación puede ser consecuencia de una recaída, lo cual significa que no se erradicó la cepa infecciosa (75\%), o que de nuevo hay infección por la exposición a una nueva cepa (25\%). El riesgo de recurrencia se asocia con la elección, la duración y el cumplimiento del tratamiento antimicrobiano (26).

Desde el punto de vista del diagnóstico, la prueba de referencia para la melioidosis es el aislamiento de B. pseudomallei a partir de especímenes clínicos; sin embargo, el microorganismo no siempre se puede aislar fácilmente, por lo que se recomienda emplear medios selectivos como el agar de Ashdown (el cual no se usa de forma rutinaria y no se encuentra disponible comercialmente); además, el tiempo de incubación es prolongado (5 a 7 días) (27).

Recientemente, se han descrito nuevas técnicas para la detección rápida y barata de la bacteria, que facilitan su uso en aquellos lugares que no cuentan con la infraestructura necesaria. Entre estas, se cuenta la detección del antígeno mediante inmunocromatografía, cuyos resultados, a pesar de ser promisorios, deben validarse $(28,29)$

Además, aunque se logre el aislamiento, no necesariamente se puede identificar adecuadamente el microorganismo. En cuanto a su fenotipo, en $B$. pseudomallei este puede identificarse utilizando sistemas comerciales. Sin embargo, estos puede que no distingan $B$. pseudomallei de $B$. thailandensis y de miembros del complejo B. cepacia (30-32) (figura 2). Asimismo, se han descrito variaciones regionales en la identificación de B. pseudomallei cuando se utilizan estos sistemas automatizados, probablemente como consecuencia del tipo de tarjetas y bases de datos empleadas (33). Entre estos métodos fenotípicos automatizados, el de mejor rendimiento diagnóstico es el Vitek $2^{\mathrm{TM}}$. EI MALDI-TOF MS ha demostrado ser un buen método diagnóstico si se utilizan las bases de datos adecuadas $(34,35)$. Para un diagnóstico más preciso se deben emplear métodos genotípicos basados en la secuenciación del rARN 16s y del gen GroER (30).

El tratamiento de esta enfermedad es prolongado. En una primera fase, se hace la inducción con ceftazidima, imipenem o meropenem intravenosos durante 10 a 14 días y, en la fase de erradicación, se emplea la administración oral de trimetoprim-suffametoxazol, solos o en combinación con doxiciclina (36). 


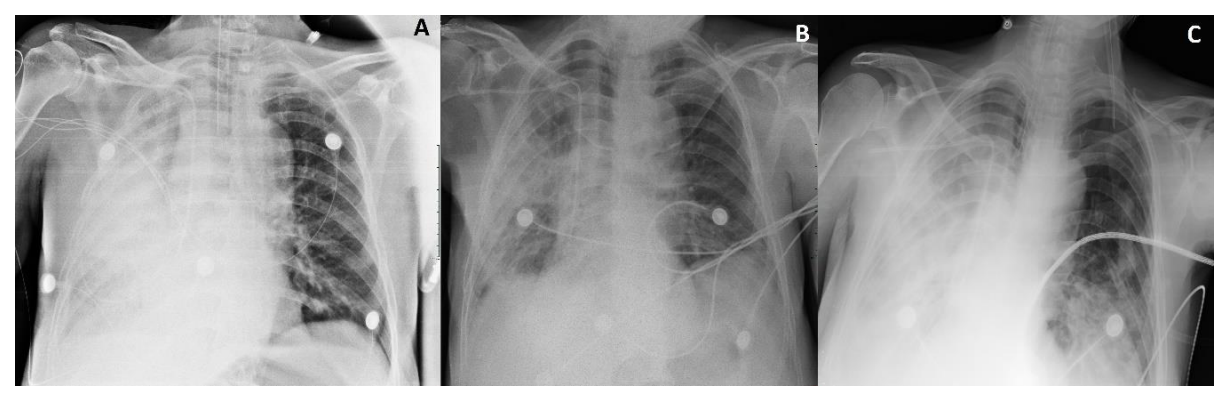

Figura 2. Radiografías de tórax. A. En el momento del ingreso. Se observan infiltrados alveolares predominantemente en el hemitórax derecho. B. En el día 15 de la estancia hospitalaria, en la que se observa disminución de los infiltrados alveolares. C. Progresión de los infiltrados alveolares en ambos campos pulmonares.

Dada la gravedad y la alta mortalidad de la enfermedad (30 a $47 \%$ en la melioidosis aguda grave y 40 a $75 \%$ en casos con sepsis) (20), así como la duración prolongada del tratamiento antimicrobiano y el escaso número de antibióticos disponibles para tratarla (37), es necesario fortalecer la vigilancia epidemiológica y la capacidad de sospecha clínica para instaurar el tratamiento más oportuno tempranamente y mejorar el resultado clínico.

Asimismo, debe contarse con herramientas diagnósticas sencillas y rápidas, como las ya descritas (por ejemplo, la inmunocromatografía), las cuales permiten un diagnóstico adecuado, especialmente en zonas potencialmente endémicas. Además, se deben fortalecer los laboratorios regionales proveyéndolos de métodos de aislamiento e identificación molecular para este germen.

Con base en los casos reportados previamente y el presentado en este estudio, y dadas las condiciones medioambientales similares a las descritas por Limmathurotsakul, et al. (2) es necesaria la búsqueda activa de casos en otras zonas del país, como los departamentos de Chocó, Santander y los de los Llanos Orientales.

Debe anotarse que cuando no se hace un diagnóstico oportuno por la falta de sospecha clínica y se prescribe el tratamiento antibiótico empírico para la presentación más usual, es decir, la neumonía adquirida en la comunidad, este resulta ineficaz, porque la recomendación actual para el manejo de primera línea de la neumonía en Colombia es el uso de ampicilina y sulbactam más claritromicina (38).

En conclusión, ante la presencia de este microorganismo en la región, se debe sospechar melioidosis en pacientes que presenten un cuadro clínico sugestivo de melioidosis con factores de riesgo, como contacto con el suelo o el agua, residencia en zonas agrícolas, trabajo en el sector de la construcción, personal militar, viajes de aventura y ecoturismo, o condiciones como diabetes mellitus, enfermedad pulmonar crónica, enfermedad renal crónica, consumo excesivo de alcohol, talasemia, tratamiento con esteroides y cáncer (36).

Los aislamientos microbiológicos de $B$. pseudomallei u otros miembros del género Burkholderia, se deben estudiar mediante más de un método de identificación microbiológica, preferiblemente los moleculares (especialmente, los sistemas Phoenix y MALDI TOF).

Por último, es necesario establecer un sistema de vigilancia activa, sobre todo en aquellas regiones de Colombia con condiciones medioambientales que favorecen la aparición de la enfermedad. 


\section{Referencias}

1. Benoit TJ, Blaney DD, Doker TJ, Gee JE, Elrod MG, Rolim DB, et al. A review of melioidosis cases in the Americas. Am J Trop Med Hyg. 2015;93:1134-9. https://doi.org/ 10.4269/ajtmh.15-0405

2. Limmathurotsakul D, Golding N, Dance DA, Messina JP, Pigott DM, Moyes CL, et al. Predicted global distribution of Burkholderia pseudomallei and burden of melioidosis. Nat Microbiol. 2016;1:15008. https://doi.org/10.1038/nmicrobiol.2015.8

3. Yabuuchi E, Kosako Y, Oyaizu H, Yano I, Hotta H, Hashimoto Y, et al. Proposal of Burkholderia gen. nov. and transfer of seven species of the genus Pseudomonas homology group II to the new genus, with the type species Burkholderia cepacia (Palleroni and Holmes 1981) comb. nov. Microbiol Immunol. 1992;36:1251-75. https://doi.org/10.1111/j.1348-0421.1992.tb02129.x

4. Hemarajata P, Baghdadi JD, Hoffman R, Humphries RM. Burkholderia pseudomallei: Challenges for the clinical microbiology laboratory. J Clin Microbiol. 2016;54:2866-73. https://doi.org/10.1128/JCM.01636-16

5. Eberl L, Vandamme P. Members of the genus Burkholderia: Good and bad guys. F1000Res. 2016;5:1007. https://doi.org/10.12688/f1000research.8221.1

6. Depoorter E, Bull MJ, Peeters C, Coenye T, Vandamme P, Mahenthiralingam E. Burkholderia: An update on taxonomy and biotechnological potential as antibiotic producers. Appl Microbiol Biotechnol. 2016;100:5215-29. https://doi.org/ 10.1007/s00253-016-7520-x

7. Nasner-Posso KM, Cruz-Calderón S, Montúfar-Andrade FE, Dance DA, Rodríguez-Morales AJ. Human melioidosis reported by ProMED. Int J Infect Dis. 2015;35:103-6. https://doi.org/10.1016/j.ijid.2015.05.009

8. Chewapreecha C, Holden MT, Vehkala M, Välimäki N, Yang Z, Harris SR, et al. Global and regional dissemination and evolution of Burkholderia pseudomallei. Nat Microbiol. 2017;2:16263. https://doi.org/10.1038/nmicrobiol.2016.263

9. Currie BJ, Kaestli M. Epidemiology: A global picture of melioidosis. Nature. 2016;529:290-1. https://doi.org/10.1038/529290a

10. White NJ. Melioidosis. Lancet. 2003;361:1715-22. https://doi.org/10.1016/S0140-6736(03)13374-0

11. Victoria P. Grandes mitos de la historia de Colombia. Primera edicion. Bogotá: Grupo Planeta-Colombia; 2011.

12. Nasner-Posso KM, Cruz-Calderón S, Rodríguez-Morales AJ, Montúfar-Andrade FE. Melioidosis: una enfermedad esporádica o emergente en Colombia? Enferm Infecc Microbiol Clin. 2015;33:206-7. https://doi.org/10.1016/j.eimc.2014.10.013

13. Severiche D. Pseudomonas pseudomallei community acquired pneumonia. Revista Colombiana de Neumología. 1998;10:188-92.

14. Montúfar FE, Ochoa JE, Ortega H, Franco L, Montúfar MC, Monsalve A, et al. Melioidosis in Antioquia, Colombia: An emerging or endemic disease? A case series. Int J Infect Dis. 2015;37:50-7. https://doi.org/10.1016/i.iiid.2015.05.023

15. Gonzáles G, Mantilla W, Rada E. Pneumonia and osteomyelitis by Burkholderia pseudomallei: Report of a clinical case. Revista Med. 2009;17:146-9.

16. Osorio HE, Durán LF. Melioidosis: reporte de un caso en el departamento del Huila - Colombia. Revista Facultad de Salud RFS 2014;6:56-9.

17. Rosero $\mathrm{CH}$, Gómez G, Gutiérrez OI. Tos y disnea de seis años de evolución en un adulto joven con melioidosis pulmonar crónica. Reporte de caso. Medlab 2013;19:465-72.

18. Guzmán-Gómez L, Agudo-Bilbao M, Peiro-Callizo E, Salas C. Melioidosis importada desde Colombia a España. Enferm Infecc Microbiol Clin. 2015;33:214-6. https://doi.org/10.1016/j.eimc.2014.06.003

19. Currie BJ. Melioidosis: Evolving concepts in epidemiology, pathogenesis, and treatment. Semin Respir Crit Care Med. 2015;36:111-25. https://doi.org/10.1055/s-0034-1398389

20. Kingsley PV, Leader M, Nagodawithana NS, Tipre M, Sathiakumar N. Melioidosis in Malaysia: A review of case reports. PLoS Negl Trop Dis. 2016;10:e0005182. https://doi.org/ 10.1371/journal.pntd.0005182

21. Suputtamongkol Y, Hall AJ, Dance DA, Chaowagul W, Rajchanuvong A, Smith MD, et al. The epidemiology of melioidosis in Ubon Ratchatani, northeast Thailand. Int J Epidemiol. 1994;23:1082-90. 
22. Currie BJ, Ward L, Cheng AC. The epidemiology and clinical spectrum of melioidosis: 540 cases from the 20 year Darwin prospective study. PLoS Negl Trop Dis. 2010;4:e900. https://doi.org/10.1371/journal.pntd.0000900

23. Wuthiekanun V, Chierakul W, Langa S, Chaowagul W, Panpitpat C, Saipan P, et al. Development of antibodies to Burkholderia pseudomallei during childhood in melioidosisendemic northeast Thailand. Am J Trop Med Hyg. 2006;74:1074-5.

24. Vishnu Prasad NR, Balasubramaniam G, Karthikeyan VS, Ramesh CK, Srinivasan K. Melioidosis of chest wall masquerading as a tubercular cold abscess. J Surg Tech Case Rep. 2012;4:115-7. https://doi.org/10.4103/2006-8808.110254

25. Limmathurotsakul D, Peacock SJ. Melioidosis: A clinical overview. Br Med Bull. 2011;99:125-39. https://doi.org/10.1093/bmb/ldr007

26. Limmathurotsakul D, Chaowagul W, Chierakul W, Stepniewska K, Maharjan B, Wuthiekanun $\mathrm{V}$, et al. Risk factors for recurrent melioidosis in northeast Thailand. Clin Infect Dis. 2006;43:979-86. https://doi.org/10.1086/507632

27. Ranjan N, Ranjan KP. State of the globe: Melioidosis: Diagnostic caveats and emerging solutions. J Glob Infect Dis. 2018;10:1-2. https://doi.org/10.4103/jgid.jgid_107_17

28. Shaw T, Tellapragada C, Ke V, AuCoin DP, Mukhopadhyay C. Performance evaluation of Active Melioidosis Detect-Lateral Flow Assay (AMD-LFA) for diagnosis of melioidosis in endemic settings with limited resources. PLoS One. 2018;13:e0194595. https://doi.org/10.1371/journal.pone.0194595

29. Woods KL, Boutthasavong L, NicFhogartaigh C, Lee SJ, Davong V, AuCoin DP, et al. Evaluation of a rapid diagnostic test for the detection of Burkholderia pseudomallei in the Lao People's Democratic Republic. J Clin Microbiol. 2018;56:e02002-17. https://doi.org/10.1128/JCM.02002-17

30. Lau SKP, Sridhar S, Ho C-C, Chow W-N, Lee K-C, Lam C-W, et al. Laboratory diagnosis of melioidosis: Past, present and future. Exp Biol Med (Maywood). 2015;240:742-51. https://doi.org/ 10.1177/1535370215583801

31. Kiratisin P, Santanirand P, Chantratita N, Kaewdaeng S, Jordan JG, Cook EC, et al. Accuracy of commercial systems for identification of Burkholderia pseudomallei versus Burkholderia cepacia. Diagn Microbiol Infect Dis. 2007;59:277-81. https://doi.org/10.1016/j.diagmicrobio.2007.06.013

32. Zong Z, Wang X, Deng Y, Zhou T. Misidentification of Burkholderia pseudomallei as Burkholderia cepacia by the VITEK 2 system. J Med Microbiol. 2012;61:1483-4. https://doi.org/10.1099/jmm.0.041525-0

33. Podin Y, Kaestli M, McMahon N, Hennessy J, Ngian HU, Wong JS, et al. Reliability of automated biochemical identification of Burkholderia pseudomallei is regionally dependent. J Clin Microbiol. 2013;51:3076-8. https://doi.org/10.1128/JCM.01290-13

34. Walewski V, Méchaï F, Billard-Pomares T, Juguet W, Jauréguy F, Picard B, et al. MALDI-TOF MS contribution to diagnosis of melioidosis in a nonendemic country in three French travellers. New Microbes and New Infections. 2016;12:31-4. https://doi.org/10.1016/j.nmni.2016.04.004

35. Wang H, Chen Y-L, Teng S-H, Xu Z-P, Xu Y-C, Hsueh P-R. Evaluation of the bruker biotyper matrix-assisted laser desorption/ionization time-of-flight mass spectrometry system for identification of clinical and environmental isolates of Burkholderia pseudomallei. Front Microbiol. 2016;7:415. https://doi.org/10.3389/fmicb.2016.00415

36. Kingsley PV, Arunkumar G, Tipre M, Leader M, Sathiakumar N. Pitfalls and optimal approaches to diagnose melioidosis. Asian Pac J Trop Med. 2016;9:515-24. https://doi.org/10.1016/j.apjtm.2016.04.003

37. Rhodes KA, Schweizer HP. Antibiotic resistance in Burkholderia species. Drug Resist Updat. 2016;28:82-90. https://doi.org/10.1016/j.drup.2016.07.003

38. Montúfar FE, Varón FA, Giraldo LF, Sáenz OA, Rodríguez A, Alarcón AM, et al. Recomendaciones para el diagnóstico, tratamiento y prevención de la neumonía adquirida en la comunidad en adultos inmunocompetentes. Infectio. 2013;17(Supl.1):1-38. https://doi.org/10.1016/S0123-9392(13)70019-5 